\title{
Effect of Metformin on Anthropometric Measurements and Hormonal and Biochemical Profile in Patients with Prediabetes
}

\author{
Mustafa Safiah, ${ }^{1}$ Dana Hyassat, ${ }^{1}$ Yousef Khader $\left(\mathbb{D},{ }^{2}\right.$ Oraib Farahid, ${ }^{1}$ Anwar Batieha $\left(\mathbb{D},{ }^{2}\right.$ \\ Mohammed El-Khateeb, ${ }^{1}$ and Kamel Ajlouni ${ }^{1}{ }^{1}$ \\ ${ }^{1}$ Department of Endocrinology, The National Center for Diabetes, Endocrinology, and Genetics, The University of Jordan, \\ Amman, Jordan \\ ${ }^{2}$ Department of Public Health, Jordan University of Science and Technology (JUST), Irbid, Jordan
}

Correspondence should be addressed to Kamel Ajlouni; ajlouni@ju.edu.jo

Received 14 May 2021; Revised 7 November 2021; Accepted 9 November 2021; Published 14 December 2021

Academic Editor: Ilaria Campesi

Copyright (C) 2021 Mustafa Safiah et al. This is an open access article distributed under the Creative Commons Attribution License, which permits unrestricted use, distribution, and reproduction in any medium, provided the original work is properly cited.

\begin{abstract}
Objectives. Metformin is the most widely preferred first-line oral antidiabetic agent that results in clear benefits in blood sugar regulation and diabetes-related complications. This study is aimed at assessing the effect of metformin on anthropometric, hormonal, and biochemical parameters in patients with prediabetes or insulin resistance. Methods. A prepoststudy was conducted among 52 patients with prediabetes or insulin resistance who met the inclusion criteria. Weight, body mass index (BMI), and waist circumference were measured before and 12 months after metformin treatment. Serum concentrations of sex steroids, gonadotropins, and lipids were also assessed. Homeostasis model assessment (HOMA) index and quantitative sensitivity check (QUICKI) index scores were calculated before metformin treatment and after 12 months of use. Results. After 12 months of metformin treatment, female patients had significant reduction in weight, BMI, and waist circumference after adjusting for age. Metformin use for 12 months resulted in significant reduction in mean fasting blood glucose and HbAlc in females only. Total cholesterol decreased significantly among men only and serum HDL-C showed a significant rise among females only. Serum LDL-C and triglycerides did not change significantly in females and males. Our study did now significant changes in ACTH and cortisol levels in both females and males after metformin treatment. Metformin use resulted in significant increase in luteinizing hormone (LH) and progesterone levels in males, while it was associated with significant increase in prolactin, follicular stimulating hormone (FSH), and dehydroepiandrostenedione-sulphate (DHEA-S) levels and significant decrease in total testosterone level in females. Conclusion. Metformin treatment in females with prediabetes reduces BMI, waist circumference, fasting blood glucose, and HbA1c. The changes in the studied parameters differed significantly according to sex.
\end{abstract}

\section{Introduction}

Metformin is the most widely preferred first-line oral antidiabetic agent that results in clear benefits in blood sugar regulation and diabetes-related complications [1]. The mechanisms underlying these benefits include the improvement of insulin sensitivity in muscles and liver and the suppression of hepatic glucose production through the inhibition of glycogenolysis and gluconeogenesis. Beyond its glucose-lowering effect, metformin has been reported to decrease fatty liver disease and to reduce microvascular as well as macrovascular complications associated with type 2 diabetes $[1,2]$.
The use of metformin in women with gestational DM (GDM) resulted in less gestational weight gain and hypertension, fewer infants with macrosomia, and less neonatal hypoglycemia [3]. However, new follow-up information on offspring of mothers with GDM or polycystic ovary syndrome (PCOS) have provided conflicting results since metformin has the potential to inhibit the primary nutrient sensor in the placenta (mTOR) and mitochondrial activity resulting in relative nutrient restriction that might adversely affect fetal growth and potentially increase the risk of childhood obesity when the offspring is exposed to an obesogenic environment [3]. Insulin resistance is the beginning of many 
disorders such as PCOS, prediabetes, diabetes, and obesity in which metformin treatment is indicated. Therefore, insulin resistance is a disease that needs treatment to reduce the consequent complications related to diabetes and prediabetes [4].

In a systematic and meta-analysis review of 31 clinical trials, metformin treatment was found to increase ovulation, reduce serum androgen levels, and improve menstrual cycle in women with PCOS [5]. On the other hand, metformin theoretically with its antigrowth properties has been suggested as an adjuvant treatment for cancer [6]. In another meta-analysis of six studies, the treatment with metformin resulted in no significant change in body weight or other lipid profile criteria. This meta-analysis showed discrepant results about the influence of metformin on lipid profile as a whole. Some studies are in agreement with metformin reducing lipid profile and have reported a reduction, whereas the others have had contradictory values and results [7].

Type 2 diabetes is strongly associated with obesity, and this close relationship has led to the connotation "diabesity," indicating the fact that the majority of individuals with type 2 diabetes are either obese or overweight. Obesity and dyslipidemia in people with type 2 diabetes are two major risk factors for coronary artery disease (CAD) and its related mortality [8]. Studies have reported that metformin is associated with reduced triglyceride (TG) and low-density lipoprotein cholesterol (LDL-C) and increased high-density lipoprotein cholesterol (HDL-C) levels [9]. It has also been reported that metformin increases nitric oxide production and can reduce the advanced glycation end products, intracellular reactive oxygen species production, and soluble vascular and intercellular cell-adhesion molecules in patients with type 2 diabetes independently of its glucose-lowering effect. As a result of all these functions, metformin could contribute to a lower risk of heart disease and mortality associated with its use $[10,11]$. This study is aimed at assessing the effect of metformin on anthropometric, hormonal, and biochemical parameters in patients with prediabetes or insulin resistance.

\section{Methods}

2.1. Study Design. A prepostdesign was performed among patients with prediabetes or insulin resistance attending the National Center for Diabetes, Endocrinology, and Genetics (NCDEG). A total of 87 consecutive patients with prediabetes and/or insulin resistance not on metformin treatment and with normal renal and liver function patients were invited to participate in this study. The exclusion criteria included congestive heart failure, kidney or liver impairment, diabetes or its complications, and pregnant or lactating women. A total of 52 patients met the inclusion/ exclusion criteria. Thirty eight of patients were females of whom 20 were postmenopausal, and 18 were premenopausal. Fourteen participants were males. The study variables were assessed and measured before administration of metformin and 12 months after the use of metformin (total dose was $1500 \mathrm{mg}$ in $53.8 \%$ of patients and $2550 \mathrm{mg}$ in $46.2 \%$ of patients). The study was approved by the ethics committee of NCDEG. All patients signed a consent form before entering the study.

2.2. Study Variables. Weight, height, and waist circumference were measured while the subjects were wearing light clothing and no shoes. Waist circumference was measured using a nonstretchable tape midway between the iliac crest and lower rib margin. Body mass index (BMI) was calculated by dividing weight $(\mathrm{kg})$ by height in meters squared $\left(\mathrm{m}^{2}\right)$.

Prediabetes was diagnosed using American Diabetes Association diagnostic criteria (2015) if the patient had fasting plasma glucose between 100 and $125 \mathrm{mg} / \mathrm{dL}$ or if the patient had impaired glucose tolerance $(140-199 \mathrm{mg} / \mathrm{dL})$ or if the patients had hemoglobin A1c (HbA1c) between 5.7\% and $6.4 \%[12]$.

Fasting blood samples were drawn from a cannula inserted into the antecubital vein from each subject before the initiation of treatment with metformin and 12 months after treatment. For the menstruating women, the blood specimens were obtained in days 2 to 5 of the cycle. All biochemical measurements (luteinizing hormone [LH], folliclestimulating hormone [FSH], thyroid-stimulating hormone [TSH], prolactin, estradiol, progesterone, total testosterone, free testosterone, sex hormone-binding globulin [SHBG], 17-OH-progesterone, dehydro-epiandrostenedione-sulphate [DHEA-S], androstenedione, cortisol, adrenocorticotropic hormone $[\mathrm{ACTH}]$, fasting insulin, FBS, high density lipoprotein cholesterol (HDL-C), low density lipoprotein cholesterol (LDL-C), total cholesterol, and triglycerides (TGs) were performed at the baseline and after 12 months of metformine use.

Fasting Homeostatic Modal Assessment (HOMA) index was calculated as (fasting insulin $(\mathrm{mU} / \mathrm{mL}) \times$ fasting glucose $(\mathrm{mg} / \mathrm{dL})$ /405). Quantitative Insulin Sensitivity Check (QUICKI) index was calculated as $1 / \log$ (fasting insulin (mU $/ \mathrm{mL})+$ fasting blood sugar $(\mathrm{mg} / \mathrm{dl})$ )

Serum concentration of glucose, total cholesterol, HDLC, LDL-C, TGs, SHBG, DHEAS, and ACTH was assayed using cobas E-6000 (Roche Diagnostics, Manheim, Germany). HbAlc was measured using dedicated high pressure liquid chromatography (HPLC) method using D10 manufactured by BioRad (Bio-Rad Laboratories, Inc.USA). Serum insulin, FT4, TSH, LH, FSH, cortisol, prolactin, estradiol, and total testosterone were assayed using the ADVIA Centaur XPT Immunoassay System (Siemens Healthcare GmbH Erlangen Germany). Androstenedione, 17-OH-progesterone, and free testosterone were assayed using ELISA (Bio-Rad, Hercules, CA, USA). All measurements were performed according to the manufacturer instructions.

2.3. Statistical Analysis. The Statistical Package for Social Science (SPSS version 20) was used for data analysis. The chi-square test was used to analyze the differences between proportions. The general linear model repeated measures analysis was used to model changes in the studied parameters after adjusting for age and waist circumference. The changes in anthropometric measures for each sex were 
adjusted for age. The changes in other parameters were adjusted for age and baseline waist circumference. A $p$ value of less than 0.05 was considered statistically significant.

\section{Results}

3.1. Participants' Characteristics. This study included 52 patients (38 females and 14 males) aged between 16 and 70 years with a mean (SD) age of 47.1 (11.7) year for females and 45.5 (7.7) year for males. The participants' characteristics are presented in Table 1 . About $79 \%$ of males and $66 \%$ of females were obese, and $14.3 \%$ of males and $25 \%$ of females were overweight. About $55 \%$ of females and $57 \%$ of males had hypertension and $42 \%$ of females and $36 \%$ males had dyslipidemia.

\subsection{Effect of Metformin Treatment on Anthropometric} Measurements. The changes in anthropometric measurements differed significantly according to sex. The changes of these parameters after 12 months of metformin were not statistically significant in males after adjusting for age. However, the measurements changed significantly among females. After 12 months of metformin treatment, female patients had significant reduction in weight (from 82.5 to $77.6 \mathrm{~kg}$, $p$ value $<0.001$ ), BMI (from 32.8 to $31.0 \mathrm{Kg} / \mathrm{m}^{2}$, $p$ value $=0.001$ ), and waist circumference $(95.5$ to $90.7 \mathrm{~cm}$, $p$ value $=0.001)$ after adjusting for age (Table 2 ).

3.3. Effect of Metformin Treatment on FBS, HbA1c, and Insulin Resistance. Metformin use for 12 months resulted in significant reduction in mean fasting blood glucose (from 101.7 to $84.1 \mathrm{mg} / \mathrm{dl}, p$ value $=0.017$ ) and HbAlc (from $5.6 \%$ to $5.4 \%$, $p$ value $=0.001)$ in females only after adjusting for age and baseline waist circumference. Insulin resistance measured by homeostasis model assessment (HOMA) index and insulin sensitivity using the quantitative sensitivity check index (QUICKI) did not change significantly in both females and males (Table 2).

3.4. Effect of Metformin Treatment on Lipid Profile. Total cholesterol decreased significantly (from 196.1 to $186.3 \mathrm{mg}$ / $\mathrm{dl}$; $p$ value $=0.028$ ) among men only, and serum HDL-C showed a significant rise after 12 months of metformin use (from 29.8 to $46.8 .0 \mathrm{mg} / \mathrm{dl}$; $p$ value $<0.001$ ) among females only after adjusting for age and baseline waist circumference (Table 2). Serum LDL-C and triglycerides did not change significantly in females and males.

3.5. Effect of Metformin on Hormonal Profile. After 12 months of metformin treatment, there was a statistically significant increase in mean $\mathrm{LH}$ and progesterone levels in males (Table 3). Metformin treatment in premenopausal women resulted in a statistically significant increase in prolactin level $(p$ value $=0.033)$ and FSH level $(p$ value $=0.022)$ as well as DHEAS level ( $p$ value $=0.015$ ) and a statistically significant decrease in total testosterone ( $p$ value $=0.032$ ).

In postmenopausal women, we noticed a significant increase in prolactin level $(p$ value $=0.043)$, LH levels $(p$ value $=0.022)$, and FSH $(p$ value $=0.008)$ as well as
TABLE 1: Baseline characteristics of study participants.

\begin{tabular}{lcc}
\hline Variable & $\begin{array}{c}\text { Female }(n=38), \\
n(\%)\end{array}$ & $\begin{array}{c}\text { Male }(n=14), \\
n(\%)\end{array}$ \\
\hline Age (years) (mean \pm SD) & $47.1 \pm 11.7$ & $45.5 \pm 7.7$ \\
Marital status & & \\
Single & $5(13.2)$ & $1(7.1)$ \\
Married & $33(86.8)$ & $13(92.9)$ \\
Body mass index(BMI) $\left(\mathrm{kg} / \mathrm{m}^{2}\right)$ & & \\
Normal & $3(9.4)$ & $1(7.1)$ \\
Overweight & $8(25.0)$ & $2(14.3)$ \\
Obese & $21(65.6)$ & $11(78.6)$ \\
Physical activity & & \\
Active & $4(10.5)$ & $3(21.4)$ \\
Not active & $34(89.5)$ & $11(78.6)$ \\
Smoking & & \\
Current & $4(10.3)$ & $9(64.3)$ \\
Nonsmoker & $35(89.7)$ & $5(35.7)$ \\
Metformin dose & & \\
1500 mg & $21(55.3)$ & $7(50.0)$ \\
2550 mg & $17(44.7)$ & $7(50.0)$ \\
Hypertension & $21(55.3)$ & $8(57.1)$ \\
Dyslipidemia & $27(71.1)$ & $10(71.4)$ \\
On statin & $16(42.1)$ & $5(35.7)$ \\
\hline
\end{tabular}

DHEAS $(p$ value $=0.005)$ after metformin treatment (Table 4).

Our study did not show significant changes in ACTH and cortisol levels in both females and males after metformin treatment. A 12-month use of metformin resulted in a statistically significant increase in TSH level (from 2.6 to $3.2 \mu \mathrm{IU} / \mathrm{mL} ; p$ value $=0.008)$ among females only (Table 5).

\section{Discussion}

Our study showed that female patients who received metformin had a significant reduction in weight, BMI, and waist circumference. In agreement with our study, Valazquez et al. [13] reported a reduction in the mean Quetelet index $\left(\mathrm{Kg} / \mathrm{cm}^{2} \times 100\right)$ and waist hip ratio after metformin treatment. On the other hand, Kowalska et al. [14] found that 4 to 5 months of metformin treatment among obese patients with PCOS resulted in a significant reduction in BMI and percentage of body fat. One study showed that higher reduction in weight was achieved with higher doses of metformin [15]. On the other hand, other studies did not show changes in body weight after metformin therapy $[16,17]$.

Total cholesterol decreased significantly among men only, and serum HDL-C showed a significant rise after 12 months of metformin use among women. Moghetti et al. [18] found that metformin significantly increased HDL-C level in women with PCOS after 6 months. Additionally, Singh et al. [19] found that administration of metformin to 22 PCOS cases in a dose of $500 \mathrm{mg}$ three times daily for 3 months was associated with a significant rise in HDL-C $(p<0.001)$, with all other lipid parameters showing no 
TABLE 2: The effect of metformin on metabolic profile in prediabetic participants.

\begin{tabular}{|c|c|c|c|}
\hline Variable & $\begin{array}{c}\text { Baseline } \\
\text { Mean } \pm \text { SD }\end{array}$ & $\begin{array}{c}\text { After } 12 \text { months } \\
\text { Mean } \pm \text { SD }\end{array}$ & Adjusted $p$ value $(\mathrm{GLM})^{*}$ \\
\hline \multicolumn{4}{|c|}{ Weight (kg) } \\
\hline Female & $82.5 \pm 14.0$ & $77.6 \pm 13.4$ & $<0.001$ \\
\hline Male & $96.3 \pm 14.5$ & $93.1 \pm 15.6$ & 0.279 \\
\hline \multicolumn{4}{|c|}{ Body mass index (BMI) } \\
\hline Female & $32.8 \pm 6.5$ & $31.0 \pm 5.9$ & 0.001 \\
\hline Male & $32.5 \pm 5.1$ & $31.6 \pm 5.6$ & 0.253 \\
\hline \multicolumn{4}{|c|}{ Waist circumference $(\mathrm{cm})$} \\
\hline Female & $95.5 \pm 10.2$ & $90.7 \pm 10.1$ & 0.001 \\
\hline Male & $105.6 \pm 10.6$ & $103.2 \pm 12.8$ & 0.290 \\
\hline \multicolumn{4}{|c|}{ Fasting blood sugar (mg/dl) } \\
\hline Female & $101.7 \pm 13.8$ & $84.1 \pm 32.8$ & 0.017 \\
\hline Male & $112.3 \pm 15.7$ & $106.0 \pm 9.0$ & 0.300 \\
\hline \multicolumn{4}{|c|}{ Hemoglobin A1c } \\
\hline Female & $5.6 \pm 0.4$ & $5.4 \pm 0.4$ & 0.001 \\
\hline Male & $5.7 \pm 0.4$ & $5.6 \pm 0.3$ & 0.041 \\
\hline \multicolumn{4}{|l|}{ Insulin } \\
\hline Female & $13.3 \pm 5.6$ & $11.8 \pm 5.4$ & 0.038 \\
\hline Male & $17.1 \pm 8.9$ & $13.6 \pm 7.8$ & 0.366 \\
\hline \multicolumn{4}{|c|}{ Quantitative Sensitivity Check (QUICKI) } \\
\hline Female & $0.3 \pm 0.02$ & $0.4 \pm 0.11$ & 0.062 \\
\hline Male & $0.3 \pm 0.03$ & $0.3 \pm 0.03$ & 0.730 \\
\hline \multicolumn{4}{|c|}{ Homeostasis model assessment (HOMA) } \\
\hline Female & $3.5 \pm 1.9$ & $3.0 \pm 1.4$ & 0.092 \\
\hline Male & $4.7 \pm 2.4$ & $3.7 \pm 2.2$ & 0.606 \\
\hline \multicolumn{4}{|c|}{ Total cholesterol } \\
\hline Female & $192.7 \pm 44.2$ & $183.9 \pm 42.4$ & 0.182 \\
\hline Male & $196.1 \pm 19.2$ & $186.3 \pm 46.1$ & 0.028 \\
\hline \multicolumn{4}{|c|}{ Low density lipoprotein cholesterol (mg/dl) } \\
\hline Female & $119.4 \pm 37.0$ & $110.5 \pm 30.2$ & 0.157 \\
\hline Male & $117.0 \pm 32.2$ & $104.2 \pm 33.8$ & 0.306 \\
\hline \multicolumn{4}{|c|}{ High-density lipoprotein cholesterol (mg/dl) } \\
\hline Female & $29.8 \pm 9.1$ & $46.8 \pm 11.9$ & $<0.001$ \\
\hline Male & $27.9 \pm 13.0$ & $40.2 \pm 12.0$ & 0.077 \\
\hline \multicolumn{4}{|c|}{ Triglyceride (mg/dl) } \\
\hline Female & $141.2 \pm 60.6$ & $133.4 \pm 62.2$ & 0.124 \\
\hline Male & $158.9 \pm 54.5$ & $152.1 \pm 53.2$ & 0.569 \\
\hline
\end{tabular}

*The changes in anthropometric variables are adjusted for age, and the changes in other variables are adjusted for age and waist circumference at the baseline. GLM: general linear model repeated measures analysis.

marked difference after metformin treatment. Moreover, Defronzo et al. [20] found that metformin treatment for 29 weeks significantly reduced plasma total cholesterol, LDLcholesterol, and TG concentration.

We found that women treated with metformin showed a significant reduction in total testosterone (but not free testosterone) and a significant increase in DHEA-S, but there was no evidence of an increase in sex hormone binding glob- ulin. The reduction in total testosterone might be partly explained by the decrease in insulin levels or by the direct effect of metformin on ovarian stroma.

Consistent with our finding, Thomas et al. [5] in a systematic review found that androgen DHEA-S was significantly increased in the metformin-treated group. Additionally, in a study conducted by Patel et al. [21] on postmenopausal women with previous PCOS and or insulin 
TABLE 3: Effect of metformin on pituitary and sex hormones in prediabetic males $(N=14)$.

\begin{tabular}{|c|c|c|c|}
\hline & Baseline, mean \pm SD & After 12 months, mean \pm SD & $p$ value \\
\hline Prolactin & $7.3 \pm 2.4$ & $7.9 \pm 2.2$ & 0.105 \\
\hline Luteinizing hormone (LH) & $3.9 \pm 1.7$ & $5.1 \pm 1.85$ & 0.003 \\
\hline Follicle-stimulating hormone (FSH) & $5.8 \pm 5.3$ & $6.2 \pm 4.3$ & 0.368 \\
\hline Free testosterone & $10.6 \pm 2.3$ & $11.5 \pm 5.0$ & 0.481 \\
\hline Total testosterone & $3.5 \pm 1.0$ & $3.7 \pm 1.4$ & 0.597 \\
\hline Estradiol & $28.0 \pm 6.6$ & $34.0 \pm 12.3$ & 0.144 \\
\hline Progesterone & $0.26 \pm 0.22$ & $0.32 \pm 0.22$ & 0.028 \\
\hline Sex hormone-binding globulin (SHBG) & $31.6 \pm 18.3$ & $31.1 \pm 14.1$ & 0.852 \\
\hline 17-OH progesterone & $2.9 \pm 1.4$ & $3.2 \pm 1.4$ & 0.068 \\
\hline Androstendione & $2.2 \pm 0.9$ & $2.3 \pm 1.1$ & 0.598 \\
\hline Dehydroepiandrostenedione-sulphate (DHEAS) & $186.1 \pm 94.4$ & $198.2 \pm 103.5$ & 0.489 \\
\hline
\end{tabular}

TABLE 4: Effect of metformin on pituitary and sex hormones in menopausal and nonmenopausal prediabetic $(N=38)$.

\begin{tabular}{|c|c|c|c|c|c|c|}
\hline & \multicolumn{3}{|c|}{ Not menopausal } & \multicolumn{3}{|c|}{ Menopausal } \\
\hline & $\begin{array}{c}\text { Baseline, mean } \\
\pm S D\end{array}$ & $\begin{array}{l}\text { After } 12 \text { months, } \\
\text { mean } \pm S D\end{array}$ & $\begin{array}{c}p \\
\text { value }\end{array}$ & $\begin{array}{c}\text { Baseline, mean } \\
\pm S D\end{array}$ & $\begin{array}{l}\text { After } 12 \text { months, } \\
\text { mean } \pm \text { SD }\end{array}$ & $p$ value \\
\hline Prolactin & $8.9 \pm 4.9$ & $10.9 \pm 6.2$ & 0.033 & $6.1 \pm 2.3$ & $7.1 \pm 3.0$ & 0.043 \\
\hline Luteinizing hormone (LH) & $4.6 \pm 2.4$ & $5.2 \pm 3.1$ & 0.455 & $28.5 \pm 18.0$ & $34.8 \pm 22.7$ & 0.022 \\
\hline Follicle-stimulating hormone (FSH) & $6.2 \pm 3.3$ & $8.1 \pm 4.7$ & 0.022 & $58.8 \pm 35.2$ & $65.3 \pm 33.0$ & 0.008 \\
\hline Free testosterone & $1.3 \pm 0.6$ & $1.2 \pm 0.5$ & 0.271 & $1.2 \pm 0.4$ & $1.3 \pm 0.6$ & 0.537 \\
\hline Total testosterone & $0.5 \pm 0.2$ & $0.42 \pm 0.1$ & 0.032 & $0.5 \pm 0.4$ & $0.47 \pm 0.6$ & 0.101 \\
\hline Estradiol & $42.9 \pm 25.1$ & $37.0 \pm 17.1$ & 0.491 & $23.6 \pm 19.6$ & $21.3 \pm 15.0$ & 0.505 \\
\hline Progesterone & $0.41 \pm 0.2$ & $0.38 \pm 0.2$ & 0.548 & $0.39 \pm 0.9$ & $0.17 \pm 0.14$ & 0.303 \\
\hline $\begin{array}{l}\text { Sex hormone-binding globulin } \\
\text { (SHBG) }\end{array}$ & $46.3 \pm 22.4$ & $45.7 \pm 21$ & 0.892 & $53.7 \pm 44.2$ & $57.7 \pm 44.4$ & 0.214 \\
\hline 17-OH progesterone & $1.6 \pm 0.8$ & $1.8 \pm 1.4$ & 0.484 & $1.3 \pm 0.9$ & $1.7 \pm 1.5$ & 0.201 \\
\hline Androstendione & $1.8 \pm 0.7$ & $1.8 \pm 0.6$ & 0.859 & $1.67 \pm 0.7$ & $1.78 \pm 0.8$ & 0.529 \\
\hline $\begin{array}{l}\text { Dehydroepiandrostenedione-sulphate } \\
\text { (DHEAS) }\end{array}$ & $120.7 \pm 65.4$ & $144.6 \pm 85.8$ & 0.015 & $89.7 \pm 53.8$ & $106.8 \pm 58.3$ & 0.005 \\
\hline
\end{tabular}

TABLE 5: Impact of effect on other hormones in prediabetic participants $(N=52)$.

\begin{tabular}{|c|c|c|c|}
\hline Variable & $\begin{array}{c}\text { Baseline } \\
\text { Mean } \pm \text { SD }\end{array}$ & $\begin{array}{l}\text { After } 12 \text { months } \\
\text { Mean } \pm \text { SD }\end{array}$ & Adjusted $p$ value $(\mathrm{GLM})^{*}$ \\
\hline \multicolumn{4}{|c|}{ Adrenocorticotropic hormone (ACTH) } \\
\hline Female & $9.6 \pm 7.5$ & $14.0 \pm 10.4$ & 0.054 \\
\hline Male & $11.3 \pm 10.7$ & $12.5 \pm 6.7$ & 0.883 \\
\hline \multicolumn{4}{|l|}{ Cortisol } \\
\hline Female & $14.8 \pm 6.0$ & $14.7 \pm 5.4$ & 0.869 \\
\hline Male & $15.6 \pm 4.7$ & $15.0 \pm 4.9$ & 0.542 \\
\hline \multicolumn{4}{|c|}{ Thyroid stimulating hormone (TSH) } \\
\hline Female & $2.6 \pm 1.2$ & $3.2 \pm 1.5$ & 0.008 \\
\hline Male & $2.6 \pm 1.4$ & $2.7 \pm 1.3$ & 0.733 \\
\hline \multicolumn{4}{|c|}{ Free thyroxine (FT4) } \\
\hline Female & $16.1 \pm 2.0$ & $16.3 \pm 2.5$ & 0.311 \\
\hline Male & $15.6 \pm 2.1$ & $15.6 \pm 2.4$ & 0.876 \\
\hline
\end{tabular}

*The changes in the parameters are adjusted for age and waist circumference at the baseline. GLM: general linear model procedure. 
resistance, metformin reduced testosterone and free testosterone, but it did not modify DHEA-S. On the other hand, Jakubowicz et al. [22, 23] studies had reported a reduction in DHEA-S with metformin in contrast to the other trials. Moreover, Campagnoli et al. [24] concluded that the use of metformin resulted in a significant reduction in free testosterone with an insignificant increase in DHEA-S levels.

In another systemic review and meta-analysis, Barba et al. [25] found that metformin decreased the circulating levels of total testosterone, DHEAS, and androstenedione, along with increasing the circulating level of SHBG, but the results were not statistically significant for free testosterone.

Our study showed a significant rise in TSH level in euthyroid female patients treated with metformin for 12 months. Consistent with our finding, Diez et al. [26] also found that patients taking metformin exhibited significantly higher TSH levels than those without metformin treatment, but this significant relationship between TSH and metformin was lost when introducing some confounding variables into the model, such as goiter, BMI, macroangiopathy, and hyperlipidemia. Lupoli et al. [27] found that metformin induces a reduction in TSH levels in patients with overt and subclinical hypothyroidism, with no change in TSH levels in euthyroid patients. Fournier et al. [28] also showed that metformin's effect on lowering TSH was observed only in the group of patients with treated hypothyroidism, while there is no effect on TSH levels in euthyroid patients, as prescribed by Vigersky et al. [29].

In our study, metformin was shown to increase prolactin levels in females with a normal baseline level of prolactin. On the other hand, Krysiak et al. [30] found that metformin decreases prolactin levels only if given at high doses to patients with elevated prolactin levels. Finally, the use of metformin in our study did not demonstrate a change in ACTH level and cortisol level. However, metformin had no effects on $17 \mathrm{OH}$-progesterone, androstenedione, estradiol, SHBG, and T4 levels in both males and females enrolled in this study. The clinical significance of these findings needs further investigation.

One of the limitations of this study is the small sample size. A larger sample size is needed to assess the effects of metformin on the studied parameters.

\section{Conclusions}

Metformin treatment in females with prediabetes reduces BMI, waist circumference, fasting blood glucose and HbAlc. The changes in the studied parameters differed significantly according to sex.

\section{Data Availability}

The SPSS data used to support the findings of this study are available from the corresponding author upon request.

\section{Conflicts of Interest}

The authors declare that they have no conflicts of interest.

\section{References}

[1] R. S. Hundal, M. Krssak, S. Dufour et al., "Mechanism by which metformin reduces glucose production in type 2 diabetes," Diabetes, vol. 49, no. 12, pp. 2063-2069, 2000.

[2] Y. M. Cho and T. J. Kieffer, "New aspects of an old drug: metformin as a glucagon-like peptide 1 (GLP-1) enhancer and sensitiser," Diabetologia, vol. 54, no. 2, pp. 219-222, 2011.

[3] L. A. Barbour and D. S. Feig, "Metformin for gestational diabetes mellitus: progeny, perspective, and a personalized approach," Diabetes Care, vol. 42, no. 3, pp. 396-399, 2019.

[4] H. Lashen, "Role of metformin in the management of polycystic ovary syndrome," Therapeutic advances in endocrinology and metabolism., vol. 1, no. 3, pp. 117-128, 2010.

[5] T. Tang, J. M. Lord, R. J. Norman, E. Yasmin, and A. H. Balen, "Insulin-sensitising drugs (metformin, rosiglitazone, pioglitazone, D-chiro-inositol) for women with polycystic ovary syndrome, oligo amenorrhoea and subfertility," Cochrane Database of Systematic Reviews, vol. 4, 2009.

[6] B. Viollet, B. Guigas, N. S. Garcia, J. Leclerc, M. Foretz, and F. Andreelli, "Cellular and molecular mechanisms of metformin: an overview," Clinical Science, vol. 122, no. 6, pp. 253$270,2012$.

[7] S. W. Gillani, N. Ghayedi, P. Roosta, P. Seddigh, and O. Nasiri, "Effect of metformin on lipid profiles of type 2 diabetes mellitus: a meta-analysis of randomized controlled trials," Journal of Pharmacy \& Bioallied Sciences, vol. 13, no. 1, pp. 76-82, 2021.

[8] J. Heshmati, M. Sepidarkish, N. Namazi et al., "Impact of dietary calcium supplement on circulating lipoprotein concentrations and atherogenic indices in overweight and obese individuals: a systematic review," Journal of Dietary Supplements, vol. 16, no. 3, pp. 357-367, 2019.

[9] H. Y. Sin, J. Y. Kim, and K. H. Jung, “Total cholesterol, high density lipoprotein and triglyceride for cardiovascular disease in elderly patients treated with metformin," Archives of Pharmacal Research, vol. 34, no. 1, pp. 99-107, 2011.

[10] J. Jager, A. Kooy, P. H. Lehert et al., "Effects of short-term treatment with metformin on markers of endothelial function and inflammatory activity in type 2 diabetes mellitus: a randomized, placebo-controlled trial," Journal of Internal Medicine, vol. 257, no. 1, pp. 100-109, 2005.

[11] C. X. Zhang, S. N. Pan, R. S. Meng et al., "Metformin attenuates ventricular hypertrophy by activating the AMP-activated protein kinase-endothelial nitric oxide synthase pathway in rats," Clinical and Experimental Pharmacology and Physiology, vol. 38, no. 1, pp. 55-62, 2011.

[12] American Diabetes Association, "Standards of medical care in Diabetes-2015: Summary of Revisions," Diabetes Care, vol. 38, Supplement_1, pp. S4-S93, 2015.

[13] E. M. Velazquez, S. Mendoza, T. Hamer, F. Sosa, and C. J. Glueck, "Metformin therapy in polycystic ovary syndrome reduces hyperinsulinemia, insulin resistance, hyperandrogenemia, and systolic blood pressure, while facilitating normal menses and pregnancy," Metabolism, vol. 43, no. 5, pp. 647654, 1994.

[14] I. Kowalska, M. Kinalski, M. Straczkowski, S. Wolczyski, and I. Kinalska, "Insulin, leptin, IGF-I and insulin-dependent protein concentrations after insulin-sensitizing therapy in obese women with polycystic ovary syndrome," European Journal of Endocrinology, vol. 144, no. 5, pp. 509-515, 2001. 
[15] L. R. Harborne, N. Sattar, J. E. Norman, and R. Fleming, "Metformin and weight loss in obese women with polycystic ovary syndrome: comparison of doses," The Journal of Clinical Endocrinology \& Metabolism, vol. 90, no. 8, pp. 4593-4598, 2005.

[16] L. C. Morin-Papunen, R. M. Koivunen, A. Ruokonen, and H. K. Martikainen, "Metformin therapy improves the menstrual pattern with minimal endocrine and metabolic effects in women with polycystic ovary syndrome," Fertility and Sterility, vol. 69, no. 4, pp. 691-696, 1998.

[17] D. A. Ehrmann, M. K. Cavaghan, J. Imperial, J. Sturis, R. L. Rosenfield, and K. S. Polonsky, "Effects of metformin on insulin secretion, insulin action, and ovarian steroidogenesis in women with polycystic ovary syndrome," The Journal of Clinical Endocrinology \& Metabolism, vol. 82, no. 2, pp. 524-530, 1997.

[18] P. Moghetti, R. Castello, C. Negri et al., "Metformin effects on clinical features, endocrine and metabolic profiles, and insulin sensitivity in polycystic ovary syndrome: a randomized, double-blind, placebo-controlled 6-month trial, followed by open, long-term clinical evaluation," The Journal of Clinical Endocrinology \& Metabolism, vol. 85, pp. 139-146, 2000.

[19] B. Singh, S. Panda, R. Nanda et al., "Effect of metformin on hormonal and biochemical profile in PCOS before and after therapy," Indian Journal of Clinical Biochemistry, vol. 25, no. 4, pp. 367-370, 2010.

[20] R. A. DeFronzo and A. M. Goodman, "Efficacy of metformin in patients with non-insulin-dependent diabetes mellitus," New England Journal of Medicine, vol. 333, no. 9, pp. 541549, 1995.

[21] S. M. Patel, N. Iqbal, S. Kaul et al., "Effects of metformin and leuprolide acetate on insulin resistance and testosterone levels in nondiabetic postmenopausal women: a randomized, placebo-controlled trial," Fertility and Sterility, vol. 94, no. 6, pp. 2161-2166, 2010.

[22] D. J. Jakubowicz, M. Seppala, S. Jakubowicz, O. Rodriguezarmas, A. Rivas-santiago, H. Koistinen et al., "Insulin reduction with metformin increases luteal phase serum glycodelin and insulin-like growth factor-binding protein 1 concentrations and enhances uterine vascularity and blood flow in the polycystic ovary syndrome," Journal of Clinical Endocrinology and Metabolism, vol. 86, no. 3, pp. 1126-1133, 2001.

[23] M. Kocak, E. Caliskan, C. Simsir, and A. Haberal, "Metformin therapy improves ovulatory rates, cervical scores, and pregnancy rates in clomiphene citrate-resistant women with polycystic ovary syndrome," Fertility and Sterility, vol. 77, no. 1, pp. 101-106, 2002.

[24] C. Campagnoli, F. Berrino, E. Venturelli et al., "Metformin decreases circulating androgen and estrogen levels in nondiabetic women with breast cancer," Clinical Breast Cancer, vol. 13, no. 6, pp. 433-438, 2013.

[25] M. Barba, H. J. Schünemann, F. Sperati et al., "The effects of metformin on endogenous androgens and SHBG in women: a systematic review and meta-analysis," Clinical Endocrinology, vol. 70, no. 5, pp. 661-670, 2009.

[26] J. J. Díez and P. Iglesias, "Relationship between serum thyrotropin concentrations and metformin therapy in euthyroid patients with type 2 diabetes," Clinical Endocrinology, vol. 78, no. 4, pp. 505-511, 2013.

[27] R. Lupoli, A. di Minno, A. Tortora, P. Ambrosino, G. Arianna Lupoli, and M. N. D. di Minno, "Effects of treatment with metformin on TSH levels: a meta-analysis of literature studies,"
The Journal of Clinical Endocrinology \& Metabolism, vol. 99, no. 1, pp. E143-E148, 2014.

[28] J. P. Fournier, H. Yin, O. H. Yu, and L. Azoulay, "Metformin and low levels of thyroid-stimulating hormone in patients with type 2 diabetes mellitus," Canadian Medical Association Journal, vol. 186, no. 15, pp. 1138-1145, 2014.

[29] R. A. Vigersky, A. Filmore-Nassar, and A. R. Glass, "Thyrotropin suppression by metformin," The Journal of Clinical Endocrinology \& Metabolism, vol. 91, no. 1, pp. 225-227, 2006.

[30] R. Krysiak, K. Kowalcze, W. Szkrobka, and B. Okopien, "The effect of metformin on prolactin levels in patients with druginduced hyperprolactinemia," European Journal of Internal Medicine, vol. 30, pp. 94-98, 2016. 